\title{
SYMMETRIC EJECTION OF MASSIVE OBJECTS FROM GALACTIC NUCLEI
}

\author{
M. J. VALTONEN \\ Institute of Astronomy, Cambridge, England \\ and \\ Research Institute for Theoretical Physics, Helsinki, Finland
}

\begin{abstract}
It is shown that massive objects can be ejected symmetrically in two opposite directions from the nucleus of a giant elliptical galaxy only under special initial conditions. A way of obtaining these conditions is outlined, and the resulting picture of evolution of galactic nuclei, including a quasar or a Seyfert galaxy phase, is shown to be compatible with observations.
\end{abstract}

\section{Introduction}

It has recently been demonstrated (Saslaw et al., 1974; subsequently called SVA) that a system of three or four massive objects can in principle be ejected from the nucleus of a giant elliptical galaxy. In particular, a system of three mass points, strongly bound to each other and of similar masses, usually breaks up after a few tens of crossing times to form a close binary, with the third particle escaping from it. The third particle escape velocity $V_{s}$, relative to the centre of mass of the triple system, is distributed as shown in Figure 1 (dotted line). The units are such that the initial

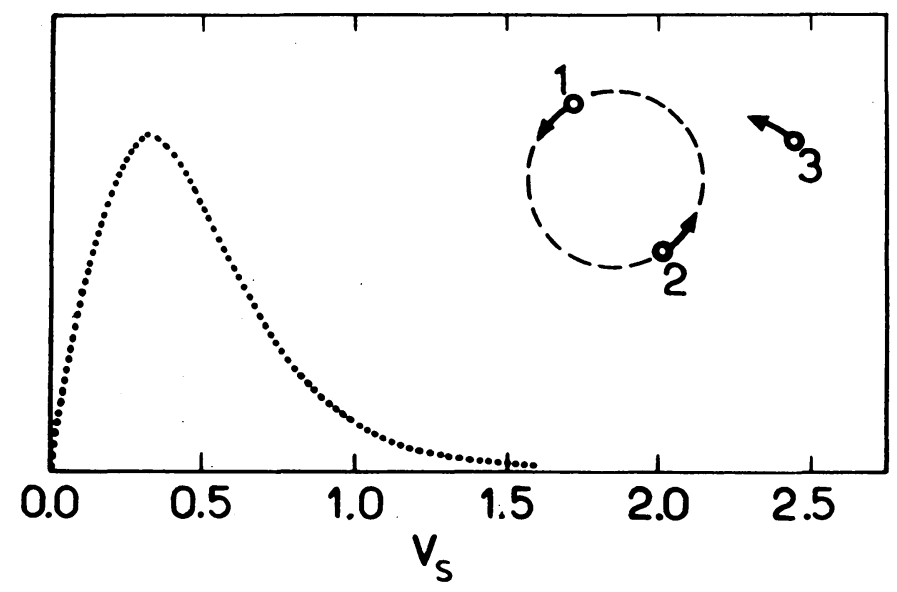

Fig. 1. The escape velocity distribution of the third particle (dotted line). The initial configuration of the three mass points is shown in the corner. For details, see the text.

binary components $m_{1}$ and $m_{2}$, which are sketched in the figure, have the velocity of 0.5 relative to their centre of mass in their circular initial orbit. This distribution is rather insensitive to the initial conditions of the system and does not depend on the mass of the escaping particle. 
The particle which escapes is usually the least massive one and a particle containing approximately half the total mass has less than $10 \%$ chance to be the escaper. Therefore the break-up is asymmetric with most of the mass in the binary, whereas the single object acquires most of the velocity. The simplest way to achieve symmetry is to keep the initial binary and the third object, with mass equal to the binary, separate throughout the interaction. We then have a binary interacting with a third particle which is initially on a nearly parabolic orbit relative to the centre of mass of the binary. In Figure 2 the average change in velocity $\Delta V$ is shown (full line) as

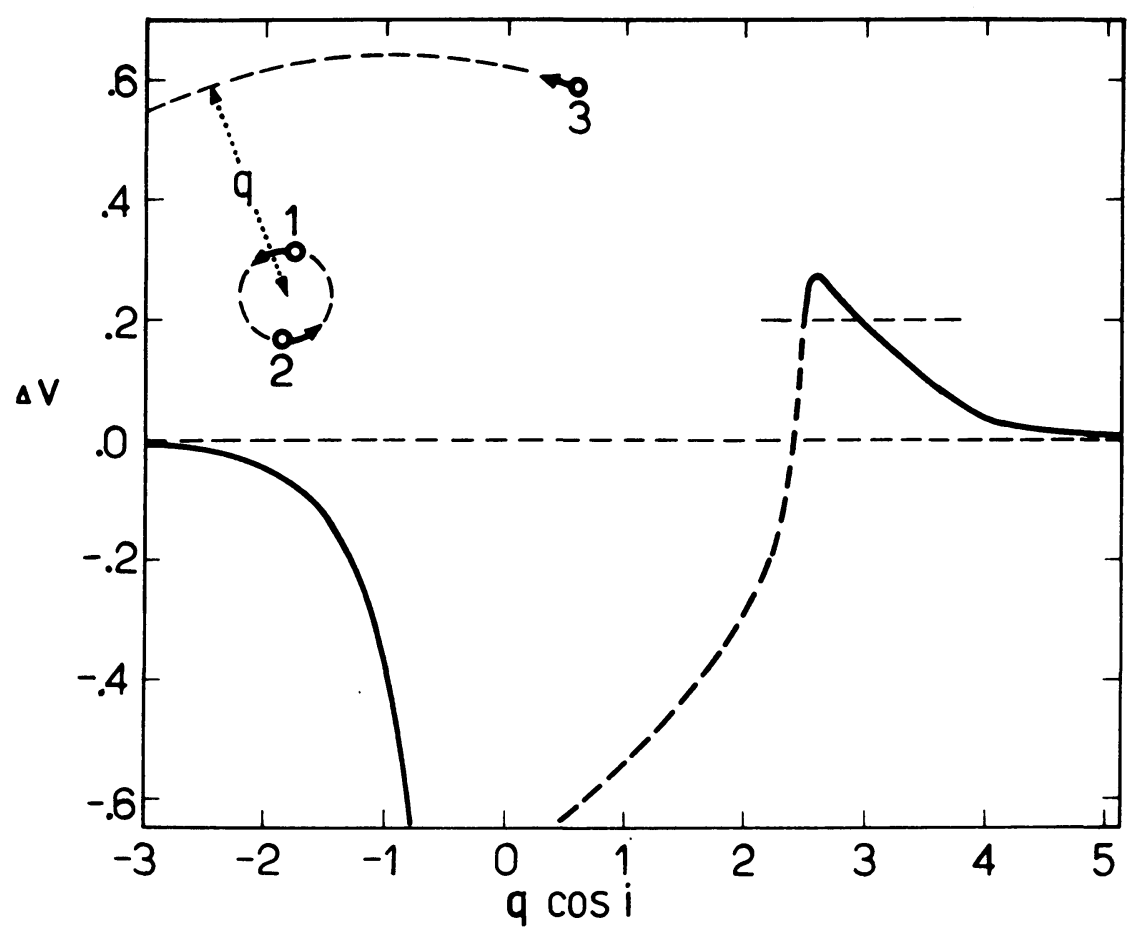

Fig. 2. The average change in the velocity of the passing particle as a function of pericentre distance $q$ for direct $(\cos i=1)$ and retrograde $(\cos i=-1)$ orbits. The initial configuration of the mass points is shown in the corner. For details, see the text.

a function of the pericentre distance $q$ of the outer orbit, which has either zero inclination relative to the binary plane (direct orbit; $\cos i=1$ ) or the inclination is $180^{\circ}$ (retrograde orbit; $\cos i=-1$ ). The average change $\Delta V$ is not well defined for $-0.8 \leqslant q$ $\times \cos i<2.5$ since the passing particle is frequently captured and becomes a component of the final binary. The semi-major axis of the outer orbit $a_{3}$ was taken to be 300, measured in terms of the semi-major axis $a$ of the initially circular binary $(e=0)$. Also $q$ is measured in units of $a$, and the velocity unit is such that the initial binary components have a velocity of 0.5 relative to their centre of mass (see the figure). It should be noted that there exists a resonance region when $q$ lies between 2.5 and 3.0 in the case of direct orbits, giving rise to a relatively large acceleration. 
The astronomical application in SVA was concerned with the ejection of radio source components, assumed to be massive compact objects $\left(\sim 10^{8} M_{\odot}\right)$, from a giant elliptical galaxy. As an example of a radio galaxy we may consider the nearby system Fornax A, for which relatively good information is available (Matthews et al., 1964; Searle, 1965). On both sides of the central object NGC 1316 there are extensive radio emitting regions, with the peak intensity areas lying on a straight line through, and approximately but not exactly equidistant from the nucleus of the galaxy. The galaxy has prominent dust arms and optical extensions lead from the galaxy near to the peak radio intensity regions. The measured rotation velocities and the geometrical configuration suggest that all the optical extensions lie in the plane perpendicular to the rotation axis of the galaxy (Searle, 1965), implying that the ejection of the radio source components has occurred in this plane. The slingshot theory (SVA) predicts preferentially this ejection direction. In the following we denote a system with this type of geometry of radio components a two-sided double.

Apparently there also exists cases when one component of the radio source lies outside the galaxy and the other coincides with the nucleus (e.g. 3C 293; Branson et al., 1972). This situation may arise through an asymmetric ejection, and we call these systems one-sided doubles. The ratio of two-sided to one-sided double radio sources is probably greater then ten. This number is rather uncertain since the present sample of well studied radio sources is small and may be biased by selection effects. There are also a great number of sources completely confined within the parent galaxy. We call these single sources irrespective of whether they show double or some other structure, since they are much too numerous to be components on their way out of the galaxy. The single and double sources are about equally common among strong radio sources. More complex sources usually show an underlying double structure which enables them to be included in the above classification scheme. A similar classification of double sources has been previously used by Miley (1971) and in that notation D2 corresponds to one-sided doubles and D1 to two-sided doubles. However, we include D1 and D2 sources in the class of single sources whenever their extent is smaller than that of the central galaxy.

\section{Dynamical Models for Radio Sources}

We have considered simple dynamical models to compare with observed radio source structures. A set of identical parent galaxies with a similar triple system of massive objects in their nuclei was assumed and the statistics of the resulting ejections was studied. A square well approximation for the galactic potential was used: any single or binary object possessing an initial velocity greater than $V_{0}\left(\sim 2000 \mathrm{~km} \mathrm{~s}^{-1}\right)$ was assumed to move outside the galaxy, whereas lower velocity objects would remain inside. When an object was ejected outside the galaxy, its distance from the nucleus $10^{9} \mathrm{yr}$ later was estimated using a more realistic galactic potential (see SVA) and assuming no interaction with the intergalactic medium.

When a triple system of three massive objects disrupts, both the single object and 
the binary receive a certain initial velocity $V$ relative to their centre of mass, taken to be the nucleus. If $V<V_{0}$ for both components, a single source is counted. When the velocity of only one component exceeds $V_{0}$, a one-sided double results and when $V>V_{0}$ for both components, a two-sided double is counted. In the latter case the ratio of the distances of the two components from the nucleus, $d_{\max } / d_{\min }$, is calculated. This classification scheme is not so artificial as it may appear. In realistic models of the galactic potential an object having a velocity $V$ only $10 \%$ below $V_{0}$ does not reach very far from the nucleus and it will return there in a relatively short time, if $V_{0}$ is properly chosen (SVA).

Two types of triple configurations were considered: a strongly bound system of three massive objects, and a third particle passing a circular binary on a direct coplanar orbit. In the former case models with various ranges in mass, total angular momentum and energy were tried, and even though this produces many different distributions, all models have a common feature: one-sided structures are by far more common than the two-sided ones. This is understandable considering the basic asymmetry of the three-body instability, and the property that the highest velocities available in such an event are not much greater than $V_{0}$ (SVA). As an example we show the distributions for one case in the second line of Table I. The mass range

TABLE I

Structure of radio sources

\begin{tabular}{llllll}
\hline & \multicolumn{2}{l}{$\begin{array}{l}\text { Two-sided doubles } \\
d_{\max } / d_{\min }\end{array}$} & & $\begin{array}{l}\text { One- } \\
\text { sided } \\
\text { doubles }\end{array}$ & $\begin{array}{l}\text { Single } \\
\text { sources }\end{array}$ \\
\cline { 1 - 3 } & $1.0-1.4$ & $1.4-3.0$ & $>3.0$ & & \\
\hline Observed & 22 & 12 & 2 & $\sim 3$ & $\sim 36$ \\
Strongly bound & 19 & 15 & 17 & $10^{3}$ & $5 \times 10^{3}$ \\
Fly-by & 34 & 0 & 27 & 60 & 120
\end{tabular}

for the three objects was taken to be within a factor of ten at most, and the physical scaling was such that no more than $50 \%$ of all triple systems would be destroyed by gravitational radiation losses before an ejection occurred (SVA). The present observations are shown in the first line of the table. The number distribution for $d_{\max } / d_{\min }$ is taken from SVA and the numbers of one-sided doubles and single sources are adjusted according to the estimates made in the previous section. The numbers in the following lines are also normalized to 34 in the first two columns. The difference between the observed and the predicted number of single sources may not be significant because single sources may evolve at a rate different from the double sources.

In the second model an equal-mass circular binary interacts with a single object of similar mass. The relative orbit of the binary centre of mass and the third object has a semi-major axis $a_{3} \simeq 200$ and pericentre distance $q$ is randomly distributed between given limits. The resulting structures again show an excess of one-sided doubles; however, these can be decreased by preventing encounters with small values of $q$. The 
third line of Table I shows an example with $q$ uniformly distributed between 0.7 and 4.0. To get distributions which correspond to the observations, within the large uncertainties of the latter, a lower limit of $q_{\min } \simeq 2.3$ must be introduced.

In conclusion, the following requirements should be satisfied by a two-sided ejection model (SVA): (1) The ejecting system must be composed of a nearly circular binary $(e \sim 0-0.05)$ and a third object weakly bound to it $\left(a_{3} / a \sim 50-300\right)$. Alternatively, the system could be made out of two approximately equal circular binaries on a weakly bound orbit with $a_{3} / a \sim 15-300$. (2) The orbits must be direct with inclination $i \sim 0^{\circ}-30^{\circ}$. The pericentre distance $q$ of the outer orbit must be greater than $\simeq 2.3$, and in most cases it should lie between 2.5 and 2.8. (3) The mass of the binary and the third object or, alternatively, the masses of the binaries, should be approximately equal: $\left(m_{1}+m_{2}\right) / m_{3} \sim 0.9-1.1$. (4) The diameter of the binary or binaries must be in range $a \sim \frac{1}{4}-2 \times 10^{-3}\left(M / 10^{8} M_{\odot}\right)$ pc, where $M=m_{1}+m_{2}$ is the binary mass. Consequently, the lifetime of the binaries due to gravitational radiation losses is $\sim 10^{2} \mathrm{yr}$ in the case of a triple system and $\sim 10^{5} \mathrm{yr}$ in the two binary systems.

\section{Formation of Massive Objects}

In principle a number of different physical processes may lead to the formation and subsequent ejection of massive objects (SVA). However, the four conditions listed in the previous section may be used to discriminate between various possibilities. The short life-time of the binary seems to exclude any scheme where the third particle is completely unrelated to the binary. The close relation between the binary and third particle masses, and the narrow range of impact distances also seem difficult to achieve in such schemes. Finally, adding the requirement of low inclinations suggests that the objects would most likely originate simultaneously in the fragmentation of a disk-like rotating object.

When a gaseous rotationally supported object cools, it flattens and, after reaching a certain critical degree of flattening, becomes dynamically unstable (Salpeter, 1971). The instability causes the disk to develop a bar-like structure which perhaps subsequently fragments into a few objects (Ostriker and Peebles, 1973). In computer simulations of a rotating disk of stars (Hohl, 1971) a bar develops with an axial ratio of about 3:2 where the length is equal to the disk diameter. It is not clear what the effect of subsequent cooling will be, but here we assume that the bar will finally fragment into two parts forming a circular binary. This may be an essential requirement from the ejection point of view since it is difficult to produce circular binaries from eccentric ones through dissipative processes within a relatively short time. We may take the semi-major axis of the resulting binary to be of the order of the radius of the initial disk.

The narrow range of impact distances and the equality of masses combined with the condition for $a_{3}$ suggests that there initially exists a very eccentric binary of two massive disks, which almost touch each other during each pericentre passage. When one or both disks fragment into a binary, an ejection may follow the next pericentre 
approach. Extremely eccentric binaries may result from an ejection of a third object from a low angular momentum planar triple system (SVA). Such a triple system may arise, for example, through an ejection of one object from a high angular momentum planar system of four bodies. The four-body system could again have been formed in a fragmentation process.

We are thus lead to consider the evolution of a rotating disk under successive stages of fragmentation. In each stage two fragments are produced, and consequently a four-body system results after two stages. Since the life-time for each generation of objects is greater than the dynamical time-scale for their mutual interactions, frequent collisions will take place, transferring angular momentum from the internal rotation of the objects into the relative motion between them (Salpeter, 1971). As a result of loosing their angular momenta the disks will shrink and may become sufficiently small to avoid suffering direct collisions any more. This would happen after contraction by a factor of $\sim 100$ (SVA). However, two of the objects may escape before this occurs. The remaining binary components might still suffer a few collisions during their first pericentre passage and shrink further until their diameters become smaller than the closest approach distance.

In Figure 3 this process is shown schematically. As an illustration we assume an

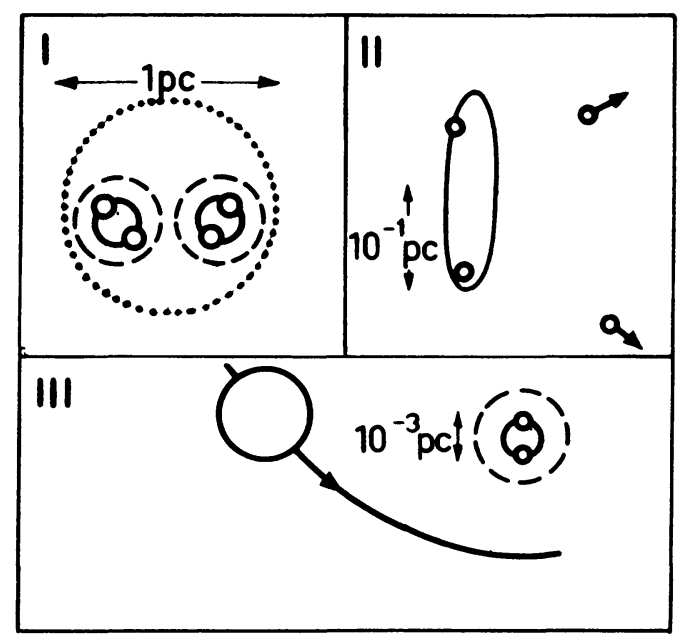

Fig. 3. A schematic representation of the three phases of the fragmentation process leading to an ejection. For details, see the text.

initial disk of diameter $1 \mathrm{pc}$ and total mass $10^{9} M_{\odot}$. In the first phase the four objects are formed and interact with each other. In the second phase only the eccentric binary remains near the nucleus while the two other objects are moving independently towards the outer parts of the galaxy. In the third phase one of the binary components fragments, and during the next pericentre passage an ejection of all three objects may occur with a speed somewhat greater than the speed of the first two ejections. 


\section{Observational Consequences}

We now briefly outline the history of a massive rotating disk in a galactic nucleus from an observational point of view. The disk could form in a time much shorter than the life-time of a galaxy from the material shed by evolving stars. It is estimated that approximately ten solar masses per year would be received by the interstellar medium of a giant elliptical galaxy from its stars (Knapp and Kerr, 1974). Due to the small specific angular momentum of a spheroidal galaxy this gas will fall to the centre of the galaxy where it will pile up and form a rotating object perhaps of $10 \mathrm{pc}$ diameter (Shklovskii, 1972). Let us suppose that $10^{10} M_{\odot}$ has collected in this object before it becomes unstable, and therefore both the length and mass scale would be of order of magnitude greater than indicated in Figure 3.

In the first phase after the instability sets in frequent collisions between the four fragments take place, each lasting $\sim 100 \mathrm{yr}$ initially but becoming shorter and more energetic later on. The total time involved in these collisions before escape takes place may not be more than $\sim 10^{3}$ yr and thus it is unlikely that we would observe the collisions occurring in any celestial object. The collisions would, however, tear off gas clouds, which would be accelerated in the gravitational field of the massive objects. The number of clouds would build up rapidly until the nuclear region is filled by them. The clouds would then collide among each other and turn their largescale kinetic energy into electromagnetic radiation through shock waves. The resulting radiation may have many of the properties observed in Seyfert galaxies (Osterbrock, 1971) and in quasi-stellar objects (Daltabuit and Cox, 1972). Also the massive objects would collide frequently with gas clouds. Such events may be observed as variable radio sources (Bignell, 1973). The fact that the best models for many variable radio sources include three or four small components (Kellermann et al., 1974) could mean that they are in their pre-ejection stage of evolution. For example in NGC 1275 the model (Legg et al., 1973) shows four components at a typical separation of a parsec from each other.

The first phase may last $\sim 10^{6} \mathrm{yr}$ after which an eccentric binary remains, and therefore a double radio structure could be observed during the second phase. The cloud collisions would be expected to cease during this phase, which may last $10^{5}-10^{6}$ yr. This is the cooling time-scale for the disks (Salpeter, 1971). After the symmetric ejection the clouds would find their gravitational binding reduced and they would start to move outwards from the nucleus. Another reminder of the past events would be a halo of relativistic particles, mainly produced during the collisions between the disks, which therefore would be elongated perpendicular to the plane of symmetry of the galaxy. These features may be observable in M87 which shows evidence of a recent symmetrical ejection (Walker and Hayes, 1967; Graham, 1970).

The ejected objects are not presumably stable themselves and may fragment again. Because of strong gravitational radiation losses, a bar instability and resulting binary formation is excluded, and only fragmentation into a large number of smaller objects seems possible. Some of the fragments could escape through three-body interactions, 
and this may be happening in one of the components of M87. One of the three main knots (perhaps the middle one) would represent the original object and the two others would be ejected objects. This interpretation may also offer an explanation of the peculiar polarization directions in the knots (Hiltner, 1959; Turland, 1975).

Some other peculiar features of M87 are worth noting. The luminous filament which connects the nucleus and the main knots originates from a point slightly north of the nucleus (Felten et al., 1970). If the filament outlines the path of the massive object from the nuclear region, it is not surprising that the path does not originate right from the middle of the nucleus, since in the present theory the eccentric binary should have been displaced by earlier ejections. Also the counterjet (Arp, 1967) and the orthogonal fan-jet (Walker, 1968) may result from interactions between gas clouds and the two independently ejected objects. The peculiar optical and radio extensions originating from the outermost bright knot could also be understood as resulting from further fragmentations and ejections, which have occurred in the ejected knots themselves.

In conclusion, the requirement for a symmetric ejection of massive objects to occur leads to a rather definite picture of the evolution of galactic nuclei which appears to have many connections with the observed forms of their activity.

\section{Acknowledgement}

We would like to thank Drs D. Lynden-Bell, J. Bardeen and F. Hohl for discussions on the fragmentation process, and Drs S. J. Aarseth, W. C. Saslaw and N. Sanitt for reading the manuscript and for valuable comments. The author has been supported by an Osk. Huttusen Säätiö-Foundation Fellowship during this work.

\section{References}

Arp, H. C.: 1967, Astrophys. Letters 1, 1.

Bignell, R. C.: 1973, Astron. J. 78, 557.

Branson, N. J. B. A., Elsmore, B., Pooley, G. G., and Ryle, M.: 1972, Monthly Notices Roy. Astron. Soc. 156, 377.

Daltabuit, E. and Cox, D.: 1972, Astrophys. J. Letters 173, L13.

Felten, J. E., Arp, H. C., and Lynds, C. R.: 1970, Astrophys. J. 159, 415.

Graham, I.: 1970, Monthly Notices Roy. Astron. Soc. 149, 319.

Hiltner, W. A., 1959, Astrophys. J. 130, 340.

Hohl, F.: 1971, Astrophys. J. 168, 343.

Kellermann, K. I., Clark, B. G., Shaffer, D. B., Cohen, M. H., Jauncey, D. L., Broderick, J. J., and Niell, A. E.: 1974, Astrophys. J. Letters, 189, L19.

Knapp, G. R. and Kerr, F. J.: 1974, Astron. J. 79, 667.

Legg, T. H., Broten, N. W., Fort, D. N., Yen, J. L., Bale, F. V., Barber, P. C., and Quigley, M. J. S. : 1973, Nature Phys. Sci. 244, 18.

Matthews, T. A., Morgan, W. W., and Schmidt, M.: 1964, Astrophys. J. $140,35$.

Miley, G. K.: 1971, Monthly Notices Roy. Astron. Soc. 152, 477.

Osterbrock, D. E.: 1971, Pontificiae Academiae Scientiarum, Scripta Varia No. 35, p. 151.

Ostriker, J. P. and Peebles, P. J. E.: 1973, Astrophys. J. 186, 467.

Salpeter, E. E.: 1971, Nature Phys. Sci. 233, 5.

Saslaw, W. C., Valtonen, M. J., and Aarseth, S. J. : 1974, Astrophys. J. 190, 253. 
Searle, L.: 1965, Nature Phys. Sci. 207, 1282.

Shklovskii, I. S.: 1972, Soviet Astron. 16, 193.

Turland, B. D.: 1975 , in press.

Walker, M. F.: 1968, Astrophys. Letters 2, 65.

Walker, M. F. and Hayes, S.: 1967, Astrophys. J. 149, 481.

\section{DISCUSSION}

Bardeen: The size of the objects is not too much smaller than their separation. Have you considered the effects of tidal interactions on the ejection process?

Valtonen: In the binary responsible for the final double ejection, any tidal elongation of the components would be dissipated by gravitational radiation and most likely the components would contract until the tidal effects become negligible.

Baldwin: After an ejection of the kind you describe, how long might it be before the situation could arise once more?

Valtonen: In this over-simplified model two ejections occur with a lower velocity before the double ejection and the massive objects from the former events may return to the nucleus and might perhaps prevent further symmetrical ejections. 